\title{
ИССЛЕДОВАНИЕ И АНАЛИЗ МЕТОДОВ ОПРЕДЕЛЕНИЯ ЭФФЕКТИВНОСТИ ПРИМЕНЕНИЯ ТЕХНОЛОГИИ ПРОПАНТНОГО ГИДРОРАЗРЫВА ПЛАСТА
}

\author{
Галкин Владислав Игнатьевич', \\ Vgalkin@pstu.ru
}

\author{
Колтырин Артур Николаевич², \\ Artur.Koltyrin@pnn.lukoil.com \\ 1 Пермский национальный исследовательский политехнический университет, \\ Россия, 614066, г. Пермь, Комсомольский пр., 29. \\ 2 ООО «ЛУКОЙЛ-ПЕРМЬ» Филиала ООО «ЛУКОЙЛ-Инжиниринг» «ПермНИПИнефть» в г. Перми, \\ Россия, 614066, г. Пермь, ул. Советской Армии, 29.
}

\begin{abstract}
Актуальность. Большинство месторождений ООО «ЛУКОЙЛ-ПЕРМЬ» находятся на завершающей стадии разработки, и на них выполняется большое количество операций гидроразрыва пласта (ГРП). В условиях высокой выработки запасов, обводнения скважин и снижения пластового давления необходимо планировать проведение ГРП на тех скважинах, где может быть получен максимальный прирост дебитов нефти.

Цель: разработка методики прогноза дебитов нефти с использованием вероятностно-статистических методов.

Объекты: данные по использованию пропантного ГРП на карбонатном ВЗВ4 объекте Батырбайского месторождения.

Методы: анализ данных по оценке эффективности ГРП с помощью использования вероятностно-статистических методов.

Результаты. С использованием вероятностно-статистического метода построены многомерные математические модели оценки эффективности ГРП, которые позволили определить приросты дебитов нефти. Одним из основных преимуществ вероятностностатистического метода является то, что все используемые для прогноза показатели, имеющие различные размерности, были переведены в безразмерные величины, что упрощает сопоставительный анализ. Кроме этого, выполнено сравнение полученных приростов дебитов нефти с методом расчета дебита жидкости через удельный коэффициент продуктивности. Выполненный анализ показал, что разработанный вероятностно-статистический метод прогноза обеспечивает более точную сходимость с фактическими данными по приростам дебитов нефти, в отличие от результатов, полученных по методу удельного коэффициента продуктивности. Значения коэффициентов парной корреляции для вероятностно-статистического метода составили по объекту разработки В3В4 - 0,85, тогда как по методу удельной продуктивности, соответственно, - 0,15, что на статистическом уровне подтверждает более высокую эффективность предлагаемого метода.
\end{abstract}

\section{Ключевые слова:}

Гидроразрыв пласта, карбонатный коллектор, прогнозирование прироста дебита нефти, вероятность, статистический расчет, удельный коэффициент продуктивности, прогнозная выборка скважин.

\section{Введение}

На месторождениях Пермского края выполнено более 1000 скв/опер гидроразрыва пластов (ГРП) начиная с 1996 г. В работе изучен и проанализирован опыт применения пропантного ГРП на карбонатном объекте В3В4 Батырбайского месторождения. Цель работы - по известным геологопромысловым, технологическим и техническим данным ГРП (масса пропанта, концентрация пропанта, давления разрыва при ГРП и т. д.) вычислить величину прироста дебита нефти $\left(Q_{\mathrm{H}}\right.$, т/сут). В связи с тем, что на успешность ГРП оказывает влияние множество параметров, использование стандартных статистических показателей и расчетов (среднее значение, зависимости одного параметра от другого и т. п.) может не дать надежных результатов прогноза. Однако с развитием математического аппарата появилась возможность обрабатывать большие массивы данных. Появились новые инструменты, такие как: дерево решений, нейронные сети, метод опорных векторов и другие, которые все чаще находят свое приложение в различных сферах нефтяной индустрии. Нейронные сети использовались для определения забойного давления при бурении на депрессии [1], пластового давления, проницаемости и скин-фактора [2]. Поскольку различные алгоритмы имеют свои достоинства и недостатки, при задачах прогноза возникает необходимость использования несколько методов, их комплексирование. Так, гибридные модели алгоритма нечеткой логики и метода опорных векторов применялись для определения ФЕС нефтяных и газовых месторождений [3]. В работе [4] приводится метод самоорганизующихся карт Кохонена (разновидность нейросетей) для выбора скважин-кандидатов под повторные ГРП. В работах [5-7] приводятся методы случайный лес и бустинг, связанные с машинным обучением. В статье [8] приведены данные по использованию многозонного гидроразрыва пласта методами Datamining в горизонтальных скважинах. Применение инструментов прогнозирования Datamining для анализа и прогноза успешности ГРП на примере объекта БВ8 Повховского месторождения приводится в статье [9]. Методы математической статистики широко применяются для анализа и прогнозирова- 
ния различных геологических и технологических показателей [10-18].

В данной статье на примере скважин объекта разработки В3В4 Батырбайского месторождения для прогнозирования приростов дебитов нефти $\left(Q_{\mathrm{H}}, \mathrm{T} /\right.$ сут $)$ после проведения ГРП приводится разработанный авторами вероятностно-статистический метод (BCM). Значение прироста дебита нефти рассчитывалось как разница среднего значения дебита нефти за три месяца после ГРП и среднего значения дебита нефти за три месяца до ГРП:

$$
Q_{\text {н }}=Q_{\text {н. после гРп }}-Q_{\text {н. до ГРП }} \text {. }
$$

Вероятностно-статистический метод основан на том, что все используемые для прогноза показатели, имеющие различные размерности, были переведены в безразмерные величины, что упрощает сопоставительный анализ сравнения показателей с разными единицами измерения $[19,20]$. В данной работе выполняется сравнение разработанного метода с методом оценки эффективности ГРП по удельному коэффициенту продуктивности (УКП). Сравнение двух методик производится по одним и тем же данным проведения ГРП, когда в качестве обучающей выборки используются результаты проведения пропантного ГРП за период до 2016 г. (по объекту разработки В3В4 - 30 операций). Сравнение результатов прогноза эффективности ГРП производится по 29 операциям, проведенным в 2017 и 2018 гг. Схема оценки эффективности проведения ГРП через удельный коэффициент продуктивности приведена на рис. 1.

\section{Описание метода расчета дебита жидкости} через удельный коэффициент продуктивности

На рис. 1 показано, что используемый алгоритм может быть описан следующим образом:

- первоначально из скважин обучающей выборки сформировали базу с геологическими и технологическими параметрами;

- по каждой скважине вычислили коэффициент продуктивности и рассчитали удельный коэффициент продуктивности на один метр эффективной нефтенасыщенной толщины;

- с учетом данных по вскрытой эффективной нефтенасыщенной толщине в скважинах с планируемым проведением пропантного ГРП рассчитали планируемый коэффициент продуктивности. Имея данные по замерам пластового давления, выполнили расчет планируемого дебита жидкости после ГРП;

- с целью расчета прироста дебита нефти после ГРП вычислим с использованием дебита жидкости дебит нефти с помощью средней обводненности после ГРП, по скважинам, выполненным до 2016 г. Среднее значение обводненности после выполнения пропантного ГРП составило $39 \%$. Из полученного дебита нефти вычислим дебит нефти до ГРП и получим прирост дебита нефти после ГРП.

\section{Описание вероятностно-статистического метода}

Вероятностно-статистический метод как способ прогнозирования прироста дебита нефти в скважине после применения ГРП для объекта разработки B3B4 кратко может быть описан следующим обра30M:

1. На первом этапе сформировали базу данных по скважинам по приростам дебитов нефти $\left(Q_{\mathrm{H}}, \mathrm{T} /\right.$ сут) после применения технологии пропантного ГРП.

2. Выполнили разделение скважин на 2 класса: 1-й класс с приростом нефти $\left(Q_{\mathrm{H}}\right)$ более 8 т/сут технология является эффективной, 2-й класс с приростом нефти $\left(Q_{\mathrm{H}}\right)$ менее 8 т/сут - технология является малоэффективной.

3. Выполнили идентификацию набора входных переменных из множества геологических, технологических и технических параметров, которые имеют влияние на выходной показатель $Q_{\mathrm{H}}$. Геологические и технологические показатели известны до выполнения ГРП, а технические параметры ГРП определяются при выполнении самой операции ГРП. Идентифицируем основные информативные геолого-технологические и технические показатели для карбонатного объекта В3В4 путем выполнения статистического анализа показателей для каждого класса по скважинам. Информативность показателей оценивалась путем сравнения средних значений в двух классах по критерию $t_{\text {p }}$ (критерий информативности).

4. Информативными геолого-технологическими являются следующие показатели: расчлененность разреза $\left(K_{\mathrm{p}}\right.$, ед), коэффициент продуктивности $\left(K_{\text {прод }}, \mathrm{M}^{3} /\right.$ сут.МПа), коэффициент пьезопроводности $\left(\gamma, \mathrm{cm}^{2} \cdot c\right)$, коэффициент проницаемости ближней зоны пласта $\left(K_{\text {прон }}^{\text {пзп }}\right.$ мкм² $)$, коэффициент проницаемости удаленной зоны пласта $\left(K_{\text {прон }}^{\text {узп }}\right.$, мкм $\left.^{2}\right)$, пластовое давление до ГРП $\left(P_{\text {пл }}, \mathrm{MПа}\right)$, нефтенасыщенная толщина $\left(h_{\text {эф }}, \mathrm{M}\right)$, скин-эффект $(S)$, данные гамма каротажа $\left(G K\right.$, мкр/ч), глубина относительная $\left(H_{\text {огн }}, \mathrm{m}\right)$, глубина абсолютная $\left(H_{\text {абс }}, \mathrm{M}\right)$, накопленная добыча нефти по скважине $\left(Q_{\text {н.н }}\right.$, т), накопленная добыча воды по скважине $\left(Q_{\text {в.н }}\right.$, т). Информативными являются следующие технические показатели: расход смеси $\left(Q_{\mathrm{cm}}, \mathrm{m}^{3} / \mathrm{мин}\right)$, объем жидкости разрыва $\left(V_{\text {ж.3 }}, \mathrm{M}^{3}\right)$, масса пропанта $\left(M_{\text {проп }}\right.$, т) концентрация пропанта $\left(K_{\text {проп }}, \kappa \Gamma / \mathrm{M}^{3}\right)$, давление в начале процесса ГРП ( $P_{\text {нач }}, \mathrm{MПа),}$ среднее давление при выполнении самой операции ГРП $\left(P_{\text {ср }}, \mathrm{MПа),} \mathrm{давление} \mathrm{в} \mathrm{конце} \mathrm{ГРП}\right.$ $\left(P_{\text {кон }}, \mathrm{MПа),} \mathrm{объем} \mathrm{геля}\left(V_{\text {геля }}, \mathrm{M}^{3}\right)\right.$.

5. На следующем шаге по каждому информативному показателю строим вероятностные модели отнесения к классу эффективного ГРП. Методика вычисления индивидуальных вероятностных моделей по каждому из показателей состоит из пяти подэтапов: 


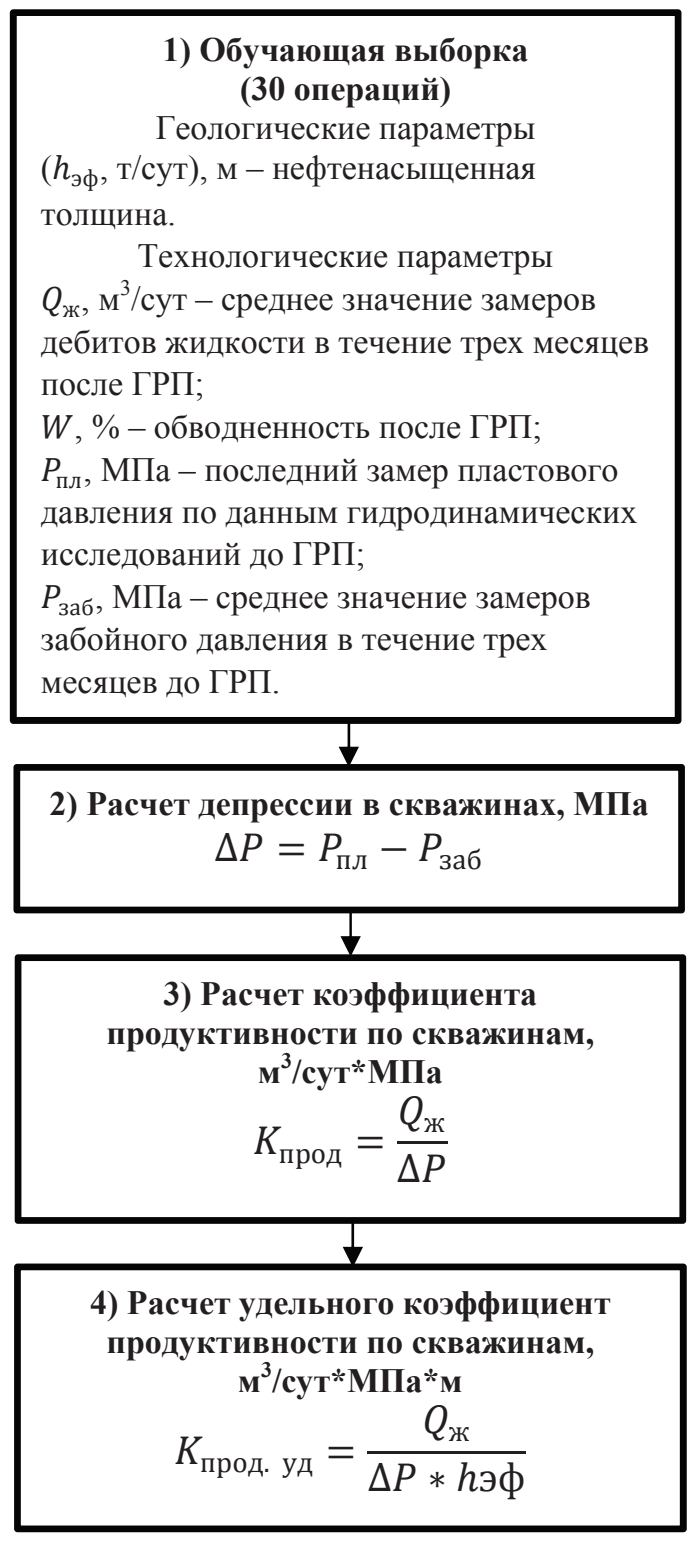

\begin{tabular}{|c|}
\hline 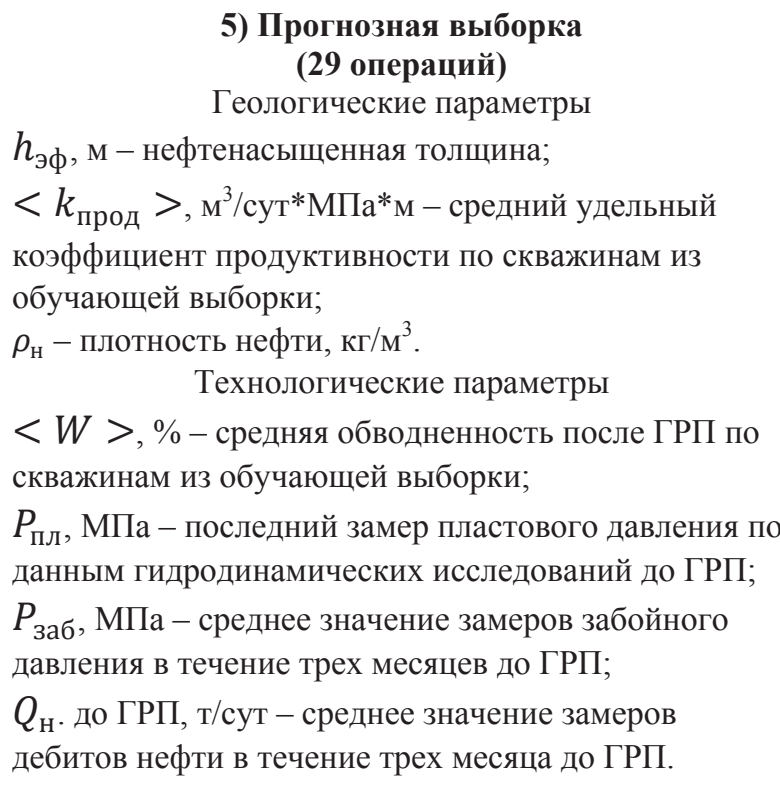 \\
\hline $\begin{array}{c}\text { 6) Расчет коэффициента продуктивности по } \\
\text { прогнозным скважинам, м³/сут МПа } \\
K_{\text {прод }}=<K_{\text {прод. уд }}>* h_{\text {эф }}\end{array}$ \\
\hline $\begin{array}{l}\text { 7) Расчет депрессии по прогнозным скважинам, } \\
\text { МПа } \\
\qquad \begin{aligned} \Delta P & =P_{\text {пл }}-P_{\text {заб }}\end{aligned}\end{array}$ \\
\hline $\begin{array}{c}\text { 8) Расчет дебита жидкости после ГРП по } \\
\text { прогнозным скважинам, } \text { м }^{3} \text { сут } \\
Q_{ж}=K_{\text {прод }} * \Delta P\end{array}$ \\
\hline $\begin{array}{l}\text { 9) Расчет дебита нефти после ГРП по прогнозным } \\
\text { скважинам, т/сут } \\
\qquad Q_{\mathrm{H.} \mathrm{после} \mathrm{грп}}=Q_{ж} * \rho_{\mathrm{H}} *<W>\end{array}$ \\
\hline $\begin{array}{c}\text { 10) Расчет прироста дебита нефти после ГРП по } \\
\text { прогнозным скважинам, т/сут } \\
Q_{\mathrm{H}}=Q_{\text {н.после грп }}-Q_{\text {н.до грп }}\end{array}$ \\
\hline
\end{tabular}

Рис. 1. Алгоритм метода расчета прироста дебита нефти

Fig 1. Algorithm of the method for calculating oil production increase

1) разделение скважин на классы по эффективности ГРП;

2) построение гистограмм по показателям для определения оптимальных величины интервалов варьирования значений;
3) вычисление вероятности принадлежности к 1 классу в каждом интервале;

4) сопоставление интервальных значений вероятности принадлежности к 1 классу со средними интервальными значениями показателя; 
Таблица 1. Вероятностные модели принадлежности к классу эффективных ГРП

Table 1. $\quad$ Probabilistic models of belonging to a class of effective hydraulic fracturing

\begin{tabular}{|c|c|c|}
\hline $\begin{array}{l}\text { Вероятностная модель } \\
\text { Probabilistic model }\end{array}$ & $\begin{array}{l}\text { Область применения } \\
\text { Application area }\end{array}$ & $\begin{array}{c}\text { Диапазон } \\
\text { изменения } \\
\text { вероятности } \\
\text { Range } \\
\text { of probability } \\
\text { change }\end{array}$ \\
\hline \multicolumn{3}{|c|}{$\begin{array}{c}\text { Геолого-технологические показатели } \\
\text { Geological and technological parameters }\end{array}$} \\
\hline$P\left(K_{\mathrm{p}}\right)=0,557-0,0329 K_{\mathrm{p}}$ & 1-3, ед. (u.f) & $0,458-0,524$ \\
\hline$P\left(K_{\text {прод }}\right)=0,491+0,0054 K_{\text {прод }}$ & $\begin{array}{c}0,21-5,68 \mathrm{~m}^{3} / \text { сут } \cdot \mathrm{MПа} \\
\left(\mathrm{m}^{3} / \mathrm{d} \cdot \mathrm{MPa}\right)\end{array}$ & $0,492-0,521$ \\
\hline 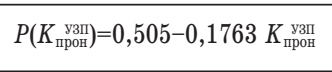 & $\begin{array}{c}0,002-0,125 \mathrm{MKM}^{2} \\
\left(\mathrm{mkm}^{2}\right)\end{array}$ & $0,482-0,504$ \\
\hline$P\left(h_{\text {эф }}\right)=0,751-0,057 h_{\text {эф }}$ & $3,0-6,5 \mathrm{~m}(\mathrm{~m})$ & $0,380-0,580$ \\
\hline$P(G K)=0,667-0,0775 G K$ & $1,4-4,0$ мкр/ч $(\mathrm{mR} / \mathrm{hr})$ & $0,357-0,558$ \\
\hline$P\left(H_{\text {абс }}\right)=-4,063+0,00528 H_{\text {абс }}$ & $848-876 \mathrm{M}(\mathrm{m})$ & $0,414-0,562$ \\
\hline$P\left(Q_{\text {в.Н }}\right)=0,468+0,000003 Q_{\text {в.Н }}$ & $90,0-46507,8 \mathrm{~T}(\mathrm{t})$ & $0,468-0,607$ \\
\hline$P(\gamma)=0,376+0,00067 \gamma$ & $14-772 \mathrm{cM}^{2} \cdot \mathrm{c}\left(\mathrm{cm}^{2} \cdot \mathrm{s}\right)$ & $0,385-0,893$ \\
\hline$P\left(K_{\text {пров }}^{\text {п3П }}=0,426+0,4299 K_{\text {прон }}^{\text {п3П }}\right.$ & $\begin{array}{c}0,0024-1,32 \text { мкм }^{2} \\
\left(\mathrm{mkm}^{2}\right)\end{array}$ & $0,427-0,993$ \\
\hline$P\left(P_{\text {пा }}\right)=0,124+0,05133 P_{\text {пл }}$ & 3,4-11,3 МПа (MPa) & $0,298-0,704$ \\
\hline$P(S)=0,447-0,0134 S$ & $-6,6-1,6$ & $0,425-0,535$ \\
\hline$P\left(H_{\text {огн }}\right)=0,225+0,00024 H_{\text {огн }}$ & $1030-1288$ м м (m) & $0,472-0,534$ \\
\hline$P\left(Q_{\text {н.Н }}\right)=0,411+0,00000474 Q_{\text {н.Н }}$ & $939,4-59862,2 \mathrm{~T}(\mathrm{t})$ & $0,415-0,650$ \\
\hline \multicolumn{3}{|c|}{ Технические показатели/Technical parameters } \\
\hline$P\left(Q_{\mathrm{cm}}\right)=-0,192+0,18094 Q_{\mathrm{cm}}$ & $\begin{array}{c}3,5-4,4 \mathrm{~m}^{3} / \mathrm{мин} \\
\left(\mathrm{m}^{3} / \mathrm{min}\right)\end{array}$ & $0,441-0,604$ \\
\hline$P\left(M_{\text {проп }}\right)=0,343+0,00578 M_{\text {проп }}$ & $19,7-36,5 \mathrm{~T}(\mathrm{t})$ & $0,456-0,553$ \\
\hline$P\left(P_{\text {нат }}\right)=0,432+0,0031 P_{\text {нач }}$ & 14-34 МПа (MPa) & $0,475-0,537$ \\
\hline$P\left(P_{\text {Кон }}\right)=0,108+0,0135 P_{\text {кон }}$ & $18-65 \mathrm{M \Pi а}(\mathrm{MPa})$ & $0,351-0,985$ \\
\hline$P\left(V_{\text {ж.p }}\right)=0,519-0,0005 V_{\text {ж.p }}$ & $12,5-70 \mathrm{M}^{3}(\mathrm{MPa})$ & $0,484-0,512$ \\
\hline$P\left(K_{\text {проп }}\right)=0,026+0,00056 K_{\text {проп }}$ & $\begin{array}{l}270-1100 \kappa \mathrm{\kappa} / \mathrm{M}^{3} \\
\left(\mathrm{~kg} / \mathrm{m}^{3}\right)\end{array}$ & $0,177-0,642$ \\
\hline$P\left(P_{\mathrm{cp}}\right)=0,313+0,0083 P_{\mathrm{cp}}$ & 14,0-30,0 МПа (MPa) & $0,429-0,532$ \\
\hline$P\left(V_{\text {геля }}\right)=0,554-0,0004 V_{\text {геля }}$ & $66,9-199,5 \mathrm{M}^{3}\left(\mathrm{~m}^{3}\right)$ & $0,474-0,527$ \\
\hline
\end{tabular}

5) вычисление парного коэффициента корреляции $r$ и построение уравнения регрессии (вероятностной модели).

Индивидуальные вероятностные модели по показателям для вычисления вероятности принадлежности к 1 классу по карбонатному объекту разработки В3В4 представлены в табл. 1.

Из табл. 2 видно, что на первом шаге при $m=2$ в

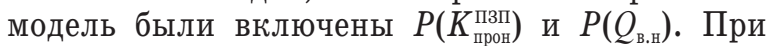
$m=3$ добавляется параметр $P\left(P_{\text {пл }}\right)$, и так далее, пока не будут привлечены все информативные показатели. Анализ $P_{\text {комп }}$ показывает, что для первого класса значения возрастают от 0,514 до 0,601. Для второго класса значения $P_{\text {ком }}$ последовательно убывают от 0,432 до 0,370 .

Таблица 3. Сочетание вероятностей технических показателей Table $3 . \quad$ Combination of probabilities of technical parameters

\begin{tabular}{|c|c|c|c|c|c|c|c|}
\hline \multirow{2}{*}{$\begin{array}{l}\text { Показатели } \\
\text { Parameters }\end{array}$} & \multicolumn{7}{|c|}{$\begin{array}{c}\text { Сочетание вероятностей }-P_{\text {комп }} \\
\text { Combination of probabilities }-P_{\text {комп }}\end{array}$} \\
\hline & $m=2$ & $m=3$ & $m=4$ & $m=5$ & $m=6$ & $m=7$ & $m=8$ \\
\hline$P\left(Q_{\mathrm{cn}}\right)$ & & + & + & + & + & + & + \\
\hline$P\left(M_{\text {проп }}\right)$ & & & + & + & + & + & + \\
\hline$P\left(P_{\text {нач) }}\right)$ & & & & & + & + & + \\
\hline$P\left(P_{\mathrm{rö}}\right)$ & + & + & + & + & + & + & + \\
\hline$P\left(V_{\text {ж. } \mathrm{p}}\right)$ & & & & & & & + \\
\hline$P\left(K_{\text {проп }}\right)$ & + & + & + & + & + & + & + \\
\hline$P\left(P_{\mathrm{cp}}\right)$ & & & & + & + & + & + \\
\hline$P\left(V_{\text {геля }}\right)$ & & & & & & + & + \\
\hline $\begin{array}{c}P_{\text {комп класс } 1} \\
P_{\text {комп }} \text { class } 1\end{array}$ & 0,536 & 0,542 & 0,545 & 0,548 & 0,549 & 0,552 & 0,551 \\
\hline \begin{tabular}{|l|}
$P_{\text {комп класс } 2}$ \\
$P_{\text {комп }}$ class 2
\end{tabular} & 0,418 & 0,416 & 0,414 & 0,413 & 0,414 & 0,415 & 0,415 \\
\hline$t_{1-2}$ & 2,753 & 2,809 & \begin{tabular}{|l|}
2,873 \\
\end{tabular} & 2,663 & 2,525 & \begin{tabular}{|l|}
2,637 \\
\end{tabular} & \begin{tabular}{|l|}
2,607 \\
\end{tabular} \\
\hline
\end{tabular}

Из табл. 3 видно, что на первом шаге при $m=2$ в модель были включены $P\left(P_{\text {кон }}\right)$ и $P\left(K_{\text {проп }}\right)$. При

Таблица 2. Сочетание вероятностей геолого-технологических показателей

Table 2. Combination of probabilities of geological and technological parameters

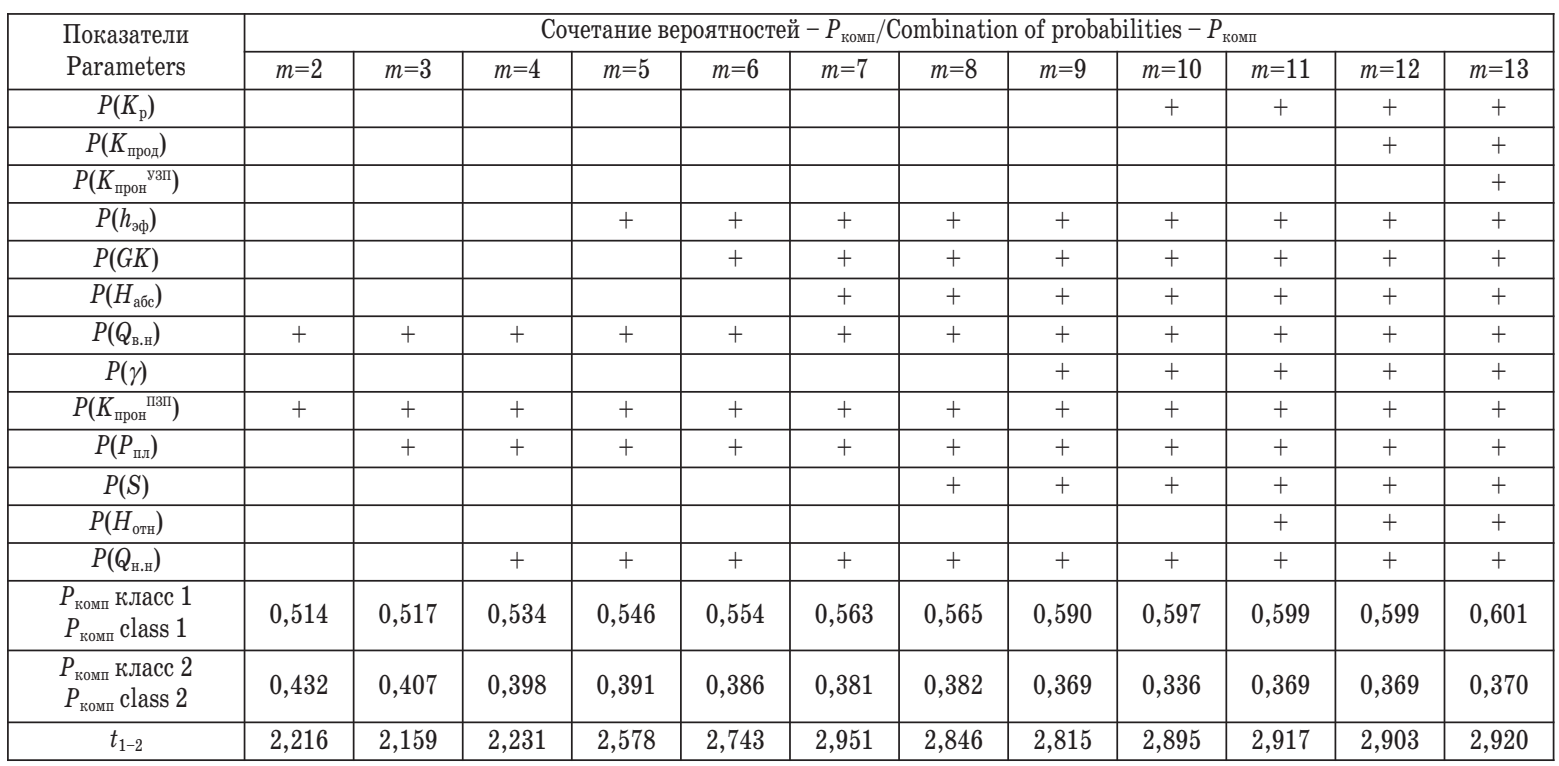


Таблица 4. Значения параметра $P_{\text {комп }}$ по скважинам при различных сочетаниях геолого-технологических вероятностей

Table 4. Values of parameter $P_{\text {комп }}$ on wells at various combinations of geological and technological probabilities

\begin{tabular}{|c|c|c|c|c|c|c|c|c|c|c|c|c|}
\hline \multirow{2}{*}{$\begin{array}{l}\text { № скв. } \\
\text { Well no. }\end{array}$} & \multicolumn{12}{|c|}{ Значения $P_{\text {комп }}$ при $m=2-13 / P_{\text {комп }}$ values at $m=2-13$} \\
\hline & $m=2$ & $m=3$ & $m=4$ & $m=5$ & $m=6$ & $m=7$ & $m=8$ & $m=9$ & $m=10$ & $m=11$ & $m=12$ & $m=13$ \\
\hline \multicolumn{13}{|c|}{ Обучающая выборка/Training selection } \\
\hline 1 & 0,729 & 0,735 & 0,878 & 0,875 & 0,932 & 0,933 & 0,943 & 0,952 & 0,918 & 0,920 & 0,945 & 0,951 \\
\hline 2 & 0,517 & 0,514 & 0,733 & 0,763 & 0,938 & 0,942 & 0,951 & 0,959 & 0,955 & 0,948 & 0,952 & 0,959 \\
\hline 3 & 0,955 & 0,958 & 0,983 & 0,986 & 0,972 & 0,974 & 0,978 & 0,982 & 0,997 & 0,997 & 0,997 & 0,997 \\
\hline \multicolumn{13}{|c|}{ Прогнозная выборка/Examination selection } \\
\hline 4 & 0,750 & 0,774 & 0,229 & 0,274 & 0,330 & 0,357 & 0,314 & 0,275 & 0,648 & 0,616 & 0,628 & 0,631 \\
\hline 5 & 0,275 & 0,293 & 0,519 & 0,572 & 0,775 & 0,795 & 0,709 & 0,605 & 0,398 & 0,424 & 0,433 & 0,471 \\
\hline 6 & 0,474 & 0,475 & 0,701 & 0,727 & 0,917 & 0,922 & 0,893 & 0,854 & 0,809 & 0,794 & 0,761 & 0,708 \\
\hline
\end{tabular}

$m=3$ добавляется параметр $P\left(Q_{\mathrm{cm}}\right)$, и так далее, пока не будут привлечены все информативные показатели. Анализ $P_{\text {комп }}$ показывает, что для первого класса значения увеличиваются незначительно от 0,534 до 0,551. Для второго класса значения

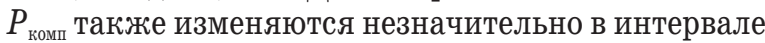
$0,418-0,413$.

На следующем шаге рассчитаем значения параметра $P_{\text {комп }}$ по каждой скважине по обучающей и прогнозной выборке при различных сочетаниях вероятностей m отдельно для геолого-технологических и технических параметров. Примеры расчета значений $P_{\text {комп }}$ по нескольким скважинам, вычисленные по геолого-технологическим показателям, приведены в табл. 4. Аналогичный расчет проводился и для технических показателей.

Из табл. 4 видно, что изменения $P_{\text {комп }}$ при различных $\mathrm{m}$ для этих скважин достаточно индивидуальны. По скв. № 6 наблюдается последовательное увеличение значений $P_{\text {комп }}$ от 0,474 до 0,708 , по скв. № 5 значения носят незакономерный характер.

Прогнозный прирост дебита нефти $Q_{\text {н }}$ рассчитаем с помощью пошагового регрессионного анализа с использованием значений $P_{\text {комп }}$ при $m$ от 2 до 13 для геолого-технологических параметров и при $\mathrm{m}$ от 2 до 8 для технических параметров. Совокупное влияние всех независимых факторов, в данном случае значений $P_{\text {комп }}$ на зависимую переменную $Q_{\text {н }}$ определим с помощью пошагового регрессионного анализа. Уравнение регрессии по геолого-технологическим показателям имеет следующий вид:

$$
Q_{\mathrm{H}}^{\mathrm{M}-\mathrm{кввГТ}}=3,629+4,987605 P_{\text {комп }_{m}^{9}} .
$$

Данная регрессионная модель учитывает значения $P_{\text {комп }}$ при сочетании $m=9$ (табл. 2), что позволяет прогнозировать прирост дебита нефти со средним отклонением от фактических значений 1,9 т/сут. На стадии подбора скважин-кандидатов рекомендуется использовать данную модель,
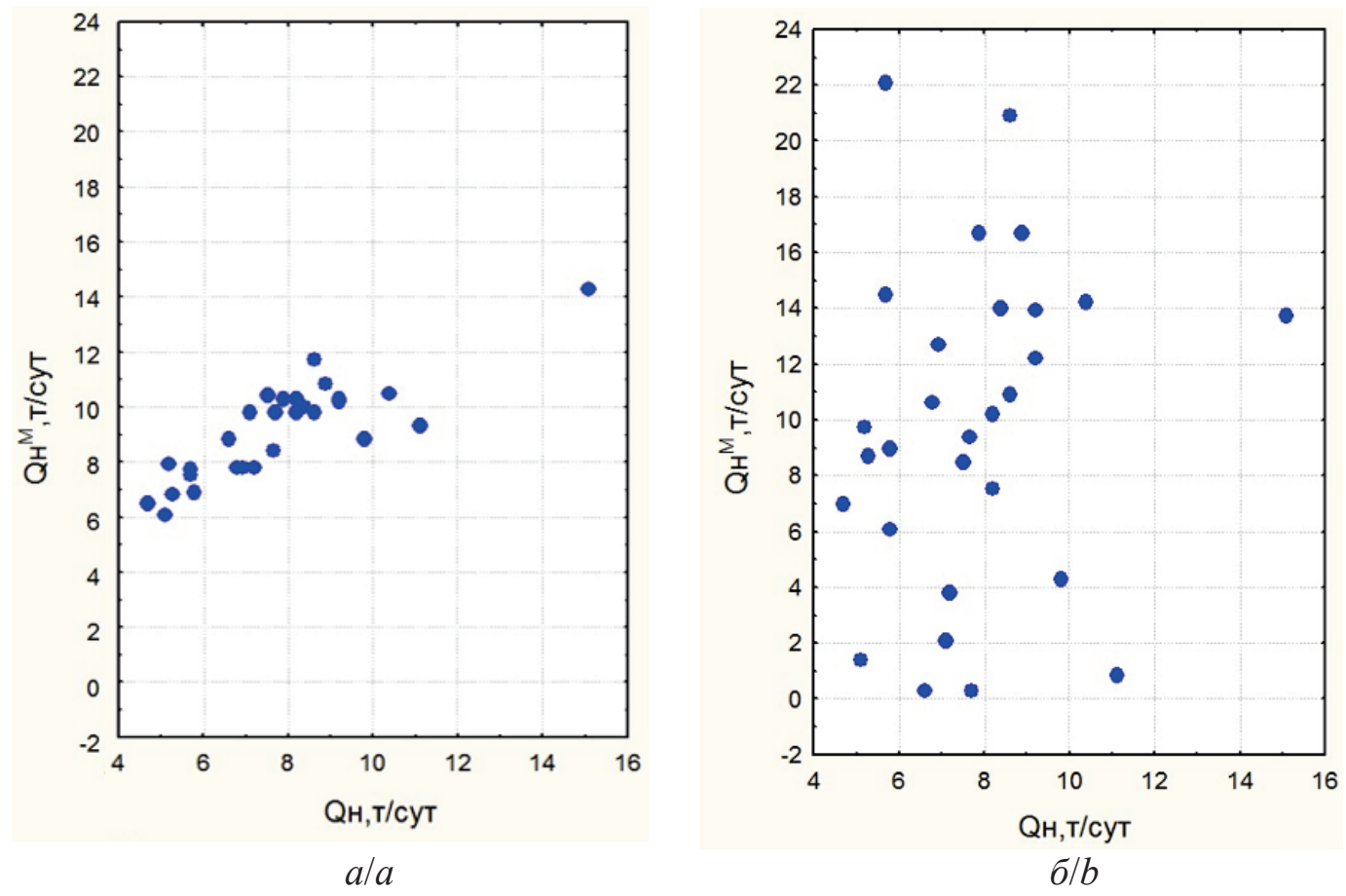

Рис. 2. Корреляиионное поле для: а) ВСМ; б) метода расчета УКП

Fig. 2. Correlation field calculated by: a) the probability-statistical method; $b$ ) the coefficient well productivity 
т. к. геолого-технологические параметры известны на стадии проектирования и, заранее имея информацию о прогнозном приросте дебита нефти, возможно ранжировать скважины по степени эффективности проведения на них ГРП.

После подбора скважины кандидата и подтверждения получение прироста по вышеприведенной модели рекомендуется выполнить операцию пропантного ГРП и по имеющейся информации о давлениях и объемах закачки реагентов выполнить расчет по модели, учитывающей только технические параметры при ГРП:

$$
\begin{aligned}
& Q_{\mathrm{H}}^{\mathrm{M}-\text { уквт }}=2,533+12,962 P_{\text {комп }_{m}^{4}}- \\
& -110,726 P_{\text {комп }_{m}^{6}}+131,161 P_{\text {комп }_{m}^{5}} .
\end{aligned}
$$

Данная регрессионная модель учитывает $P_{\text {комп }}$ при сочетании $m=4,5,6$, что позволяет прогнозировать прирост дебита нефти со средним отклонением от фактических значений 1,9 т/сут.

Для подтверждения и получения результата с наименьшей ошибкой рекомендуется выполнить расчет по следующей формуле, которая совместно учитывает геолого-технологические и технические показатели:

$$
\begin{aligned}
& Q_{\mathrm{H}}^{\mathrm{M} \text {-уквГТ-Т }}=0,786-1,0433 Q_{\mathrm{H}}^{\mathrm{M}-\mathrm{уквГТ}}+1,5429 Q_{\mathrm{H}}^{\mathrm{M}-\mathrm{укв \textrm {T }}}+ \\
& +0,277\left(Q_{\mathrm{H}}^{\mathrm{M}-\mathrm{уквГT}}\right)^{2}-0,3261 Q_{\mathrm{H}}^{\mathrm{M}-\mathrm{уквГТ}} Q_{\mathrm{H}}^{\mathrm{M}-\mathrm{укв \textrm {T }}}+ \\
& +0,1039\left(Q_{\mathrm{H}}^{\mathrm{M}-\text { уквт }}\right)^{2} \text {. }
\end{aligned}
$$

Данное уравнение позволяет прогнозировать прирост дебита нефти со средним отклонением от фактических значений 1,7 т/сут.

В работе прогнозирование прироста дебита нефти и сравнение с методом УКП будет осуществляться с использованием модели учитывающей как геолого-технологические, так и технические показатели.

\section{Результаты прогнозирования прироста дебита нефти}

Результаты расчетов по вышерассмотренным методам представлены в табл. 5 .

По данным, приведенным в табл. 5, построены корреляционные поля для двух анализируемых методик.

На рис. 2, а показано, что значения прироста дебита нефти рассчитанного ВСМ достаточно хорошо коррелируется с фактическими приростами дебитов, значение коэффициента корреляции $\mathrm{r}=0,85$, при достигаемом уровне значимости $\mathrm{p}<0,0000$. Для модели УКП (рис. 2, б) значения приростов дебитов нефти практически не коррелируются между собой, $\mathrm{r}=0,15$, при достигаемом

\begin{tabular}{|c|c|c|c|}
\hline \multirow[b]{2}{*}{$\begin{array}{l}\text { № скв. } \\
\text { Well no. }\end{array}$} & \multicolumn{3}{|c|}{$\begin{array}{c}\text { Прирост дебита нефти, т/сут } \\
\text { Increase of oil production rate, ton/day }\end{array}$} \\
\hline & $\begin{array}{l}\text { Факти- } \\
\text { ческий } \\
\text { Actual }\end{array}$ & $\begin{array}{l}\text { Рассчитанный через } \\
\text { удельный коэффици- } \\
\text { ент продуктивности } \\
\text { Calculated by the coeffi- } \\
\text { cient well productivity }\end{array}$ & $\begin{array}{c}\text { Рассчитанный вероят- } \\
\text { ностно-статистическим } \\
\text { методом } \\
\text { Calculated by the proba- } \\
\text { bility-statistical method }\end{array}$ \\
\hline 1 & 4,7 & 7,0 & 6,5 \\
\hline 2 & 5,1 & 1,4 & 6,1 \\
\hline 3 & 5,2 & 9,7 & 7,9 \\
\hline 4 & 5,3 & 8,7 & 6,8 \\
\hline 5 & 5,7 & 22,1 & 7,7 \\
\hline 6 & 5,7 & 14,5 & 7,5 \\
\hline 7 & 5,8 & 6,1 & 6,9 \\
\hline 8 & 5,8 & 9,0 & $\begin{array}{ll}6,9 \\
\end{array}$ \\
\hline 9 & 6,6 & 0,3 & 8,8 \\
\hline 10 & 6,8 & 10,6 & 7,8 \\
\hline 11 & 6,9 & 12,7 & 7,8 \\
\hline 12 & 7,1 & 2,1 & 9,8 \\
\hline 13 & 7,2 & 3,8 & 7,8 \\
\hline 14 & 7,5 & 8,5 & 10,4 \\
\hline 15 & 7,6 & 9,4 & 8,4 \\
\hline 16 & 7,7 & 0,3 & 9,8 \\
\hline 17 & 7,9 & 16,7 & 10,3 \\
\hline 18 & 8,2 & 10,2 & 9,8 \\
\hline 19 & 8,2 & 7,5 & 10,3 \\
\hline 20 & 8,4 & 14,0 & 10,0 \\
\hline 21 & 8,6 & 20,9 & 9,8 \\
\hline 22 & 8,6 & 10,9 & 11,7 \\
\hline 23 & 8,9 & 16,7 & 10,8 \\
\hline 24 & 9,1 & 13,9 & 10,2 \\
\hline 25 & 9,2 & 12,2 & 10,3 \\
\hline 26 & 9,8 & 4,3 & 8,8 \\
\hline 27 & 10,4 & 14,2 & 10,5 \\
\hline 28 & 11,1 & 0,8 & 9,3 \\
\hline 29 & 15,1 & 13,7 & 14,3 \\
\hline
\end{tabular}
уровне значимости $\mathrm{p}<0,434$.
Таблииа 5. Сравнение результатов прогнозирования с фактическими значениями

Table 5. Comparison of forecasting results with the actual values

\section{Выводы}

Основные преимущества предлагаемого метода заключаются в том, что разработанный метод позволяет выполнять сравнение показателей, имеющих разные единицы измерения, использовать для прогноза неограниченное количество информативных показателей, в отличие от метода расчета удельной продуктивности, где учитывается их ограниченное число. Кроме этого, последовательное построение раздельных моделей прогноза эффективности ГРП по совокупности геолого-технологических показателей позволяет осуществлять выбор наиболее перспективных скважин для проведения ГРП, по оптимальной совокупности технических условий проведения ГРП в этих скважинах можно получить наиболее высокую его эффективность. 


\section{СПИСОК ЛИТЕРАТУРЫ}

1. Ashena R., Moghadasi J. Bottom hole pressure estimation using evolved neural networks by real coded ant colony optimization and genetic algorithm // Journal of Petroleum Science and Engineering. - 2011. - V. 77 (3-4). - P. 375-385.

2. Jeirani Z., Mohebbi A. Estimating the initial pressure, permeability and skin factor of oil reservoir using artificial neural networks. Journal of Petroleum Science and Engineering // Journal of Petroleum Science and Engineering. - 2006. - V. 50 (1). P. 11-20.

3. Anifowose F., Abdulraheem A. Fuzzy logic-driven and SVM-driven hybrid computational intelligence models applied to oil and gas reservoir characterization // Journal of Natural Gas Science and Engineering. - 2011. - V. 3 (3). - P. 505-517.

4. Oberwinkler C., Economides M. The definitive identification of candidate wells for refracturing // SPE Annual Technical Conference and Exhibition. - Denver, Colorado, 2003. - SPE 84211.

5. Classification and Regression Trees / L. Breiman, J. Friedman, C.J. Stone, R.A. Olshen. - New York: Chapman and Hall/CRC, 1984. P. 368.

6. Breiman L. Random Forests // Machine Learning. - 2001. V. 45 (1). - P. 5-32.

7. Shapire R.E., Freund Y. Boosting // Foundations and algorithms. - Cambridge: The MIT Press, 2012. - P. 544.

8. Анализ фонда горизонтальных скважин с многозонным Гидроразрывом пласта методами Datamining / B.В. Рожкова, Р.Т. Алимханов, Р.М. Сабитов, А.В. Бухаров, М.О. Воловик // Геология, геофизика и разработка нефтяных и газовых месторождений. - 2018. - № 9. - С. 84-89.

9. Алимханов Р., Самойлова И. Применение инструментов Datamining для анализа и прогноза успешности ГРП на примере объекта БВ8 Повховского месторождения // Конференция SPE по разведке и добыче. - М., Россия, 14-16 октября, 2014. - SPE-171332-RU.

10. Соснин Н.Е. Разработка статистических моделей для прогноза нефтегазоносности (на примере терригенных девонских отложений Северо-Татарского свода) // Вестник Пермского национального исследовательского политехнического университета. Геология. Нефтегазовое дело. - 2012. - № 5. - С. 16-25.

11. Галкин В.И., Соснин Н.Е. Разработка геолого-математических моделей для прогноза нефтегазоносности сложнопостроенных структур в девонских терригенных отложениях // Нефтяное хозяйство. -2013 . - № 4. - С. 28-31.
12. Андрейко С.С. Разработка математической модели метода прогнозирования газодинамических явлений по геологическим данным для условий Верхнекамского месторождения калийных солей // Вестник Пермского национального исследовательского политехнического университета. Геология. Нефтегазовое дело. - 2016. - № 21. - С. 345-353.

13. Practical Application of a Probabilistic Approach to Estimate Reserves Using Production Decline Data / Y. Cheng, Y. Wang, D. McVay, W.J. Lee // SPE Economics and Management. 2010. - V. 2 (1). - P. 1047-1057. DOI: 10.2118/95974-pa.

14. Clark A.J., Lake L.W., Patzek T.W. Production Forecasting with Logistic Growth Models // SPE Annual Technical Conference and Exhibition. - Denver, Colorado, USA, 2011. - SPE 144790-ms. DOI: $10.2118 / 144790-\mathrm{ms}$.

15. Gong X., Gonzalez R., McVay D. Bayesian Probabilistic Decline Curve Analysis Quantifies Shale Gas Reserves Uncertainty // Canadian Unconventional Resources Conference. - Alberta, Canada, 2011. SPE 147588. DOI: $10.2118 / 147588-\mathrm{ms}$

16. Mattar L. Production Analysis and Forecasting of Shale Gas Reservoirs: Case History-Based Approach // SPE Shale Gas Production Conference. - Fort Worth, Texas, USA, 2008. - SPE 119897ms. DOI: $10.2118 / 119897-\mathrm{ms}$.

17. McVay D.A., Dossary M.N. The Value of Assessing Uncertainty // SPE Journal. - 2014. - V. 6 (2). - P. 100-110. DOI: 10.2118/ 160189-pa.

18. Xin Ma, Zhibin Liu. Predicting the oil field production using the novel discrete GM $(1, \mathrm{~N})$ model // The Journal of Grey System. 2015. - № 4. - P. 63-73.

19. Галкин В.И., Казанцев А.С., Колтырин А.Н. Вероятностностатистическая оценка использования различных показателей для определения эффективности применения пропантного ГРП (на примере терригенного объекта Тл-Бб и карбонатного объекта В3В4) // Нефтепромысловое дело. - 2018. - № 2. C. 26-32.

20. Галкин В.И., Пономарева И.Н., Колтырин А.Н. Разработка вероятностно-статистических моделей для оценки эффективности применения пропантного гидравлического разрыва пласта (на примере объъекта Тл-Бб Батырбайского месторождения) // Вестник Пермского Национального Исследовательского Политехнического Университета. Геология. Нефтегазовое и горное дело. - 2018. - № 1. - С. 37-45.

Поступила 18.12.2018.

\section{Информация об авторах}

Галкин В.И., доктор геолого-минералогических наук, профессор, заведующий кафедрой геологии нефти и газа Пермского национального исследовательского политехнического университета.

Колтырин A.H., инженер I категории отдела проектирования и мониторинга методов повышения нефтеотдачи пластов месторождений 000 «ЛУКОЙЛ-ПЕРМЬ» Филиала 000 «ЛУКОЙЛ-Инжиниринг» «ПермНИПИнефть» в г. Перми. 


\title{
RESEARCH AND ANALYSIS OF METHODS FOR DETERMINING THE EFFICIENCY OF APPLICATION OF THE PROPPANT HYDRAULIC FRACTURING
}

\author{
Vladislav I. Galkin', \\ Vgalkin@pstu.ru \\ Artur N. Koltyrin², \\ Artur.Koltyrin@pnn.lukoil.com \\ 1 Perm National Research Polytechnic University, \\ 29, Komsomolskiy avenue, Perm, 614066, Russia. \\ 2 LUKOIL-PERM LLC of PermNIPIneft branch of LUKOIL-Engineering LLC in Perm, \\ 29, Sovetskoy Armii street, Perm, 614066, Russia.
}

Relevance. A great number of hydraulic fracturing treatments are performed on LUKOIL-PERM LLC fields. A lot of fields are in the final stages of development. In conditions of a high degree of recovery of reserves, high water cut and decrease in reservoir pressure it is necessary to justify target well performance indicators on the new level.

The aim of the research is to forecast the magnitude of the increase in oil production rate using the probabilistic-statistical approach and the method for computation by specific productivity index, compare the results obtained with the real data.

Objects: the effectiveness of the proppant hydraulic fracturing at the carbonate V3V4 reservoir of the Batyrbayskoe field.

Research methods: probability theory and mathematical statistics.

Results. Use of the probabilistic and statistical analysis allowed constructing multidimensional mathematical models for assessment of hydraulic fracturing efficiency. These models predicted the efficiency of hydraulic fracturing. The main advantage of a probabilistic and statistical method is: all parameters used for the forecast with various dimensions are transferred to dimensionless sizes that simplifies comparative analysis. Comparison of the received efficiency of hydraulic fracturing with a standard method of calculation of liquid output through specific coefficient of efficiency is executed. The carried out analysis showed that the developed probabilistic and statisti$\mathrm{cal}$ method of the forecast provides more exact convergence with actual data, unlike the results received by the method of efficiency specific coefficient. The obtained values of coefficients of pair correlation for the probabilistic and statistical method is 0,85 , while the ones obtained by the specific efficiency method is 0,15 . At the statistical level higher efficiency of the probabilistic and statistical method is confirmed.

\section{Key words:}

Hydraulic fracturing, carbonate reservoir, forecast of oil production increase, probability, statistical computation, specific productivity index, test selection of wells.

\section{REFERENCES}

1. Ashena R., Moghadasi J. Bottom hole pressure estimation using evolved neural networks by real coded ant colony optimization and genetic algorithm. Journal of Petroleum Science and Engineering, 2011, vol. 77 (3-4), pp. 375-385.

2. Jeirani Z., Mohebbi A. Estimating the initial pressure, permeability and skin factor of oil reservoir using artificial neural networks. Journal of Petroleum Science and Engineering, 2006, vol. 50 (1), pp. 11-20.

3. Anifowose F., Abdulraheem A. Fuzzy logic-driven and SVM-driven hybrid computational intelligence models applied to oil and gas reservoir characterization. Journal of Natural Gas Science and Engineering, 2011, vol. 3 (3), pp. 505-517.

4. Oberwinkler C., Economides M. The definitive identification of candidate wells for refracturing. SPE Annual Technical Conference and Exhibition. Denver, Colorado, 2003. SPE 84211.

5. Breiman L., Friedman J., Stone C.J., Olshen R.A. Classification and Regression Trees. New York, Chapman and Hall/CRC, 1984. $368 \mathrm{p}$.

6. Breiman L. Random Forests. Machine Learning, 2001, vol. 45 (1), pp. $5-32$.

7. Shapire R.E., Freund Y. Boosting. Foundations and algorithms. Cambridge, The MIT Press, 2012. pp. 544.

8. Rozhkova V.V., Alimkhanov R.T., Sabitov R.M., Bukharov A.V., Volovik M.O. Analysis of horizontal wells fund with a multi-zone hydraulic fracturing of a formation by data mining methods. Geology, geophysics and development of oil and gas fields, 2018, no. 9, pp. 84-89. In Rus.
9. Alimkhanov R.T., Samoylova I. Use of the Data mining tools for the analysis and the forecast of success of hydraulic fracturing on the example of an object BV8 of the Povkhovsky field. SPE conference on exploration and production. Moscow, Russia, 14-16 0ctober, 2014. SPE-171332-RU. In Rus.

10. Sosnin N.E. Development of statistical models for predicting oiland-gas content (on the example pf terrigenous devonian sediments of North Tatar arch). Perm Journal of Petroleum and Mining Engineering, 2012, no. 5, pp. 16-25. In Rus.

11. Galkin V.I., Sosnin N.E. Geological development of mathematical models for the prediction of oil and gas complex-built structures in the Devonian clastic sediments. Oil industry, 2013, no. 4, pp. 28-31. In Rus.

12. Andreiko S.S. Development of mathematical model of gas-dynamic phenomena forecasting method according to geological data in conditions of Verkhnekamskoie potash salt deposit. Perm Journal of Petroleum and Mining Engineering, 2016, no. 21, pp. 345-353. In Rus. DOI: 10.15593/224-9923/2016.21.6.

13. Cheng Y., Wang Y., McVay D., Lee W.J. Practical Application of a Probabilistic Approach to Estimate Reserves Using Production Decline Data. SPE Economics and Management, 2010, vol. 2 (1), P. 1047-1057. DOI: 10.2118/95974-pa.

14. Clark A.J., Lake L.W., Patzek T.W. Production Forecasting with Logistic Growth Models. SPE Annual Technical Conference and Exhibition. Denver, Colorado, USA, 2011. SPE 144790-ms. DOI: 10.2118/144790-ms.

15. Gong X., Gonzalez R., McVay D. Bayesian Probabilistic Decline Curve Analysis Quantifies Shale Gas Reserves Uncertainty. Can- 
adian Unconventional Resources Conference. Alberta, Canada, 2011. SPE 147588. DOI: $10.2118 / 147588$-ms

16. Mattar L. Production Analysis and Forecasting of Shale Gas Reservoirs: Case History-Based Approach. SPE Shale Gas Production Conference. Fort Worth, Texas, USA, 2008. SPE 119897-ms. DOI: $10.2118 / 119897-\mathrm{ms}$.

17. McVay D.A., Dossary M.N. The Value of Assessing Uncertainty. SPE Journal, 2014, vol. 6 (2), pp. 100-110. DOI: 10.2118/160189-pa.

18. Xin Ma, Zhibin Liu. Predicting the oil field production using the novel discrete GM $(1, \mathrm{~N})$ model. The Journal of Grey System, 2015, no. 4, pp. 63-73.

19. Galkin V.I., Kazantsev A.S., Koltyrin A.N. Probability-statistical estimation of the use of different indicators for the determi-

\section{Information about the authors}

Vladislav I. Galkin, Dr. Sc., professor, head of the Department of Oil and Gas Geology, Perm National Research Polytechnic University.

Artur N. Koltyrin, $1^{\text {st }}$ category engineer, Department of Enhanced Oil Recovery Methods Design and Monitoring of the fields of LUKOIL-PERM LLC of PermNIPIneft branch of LUKOIL-Engineering LLC in Perm. nation of the efficiency of application of the propant fuel assembly (on the example of terrigenous object of $\mathrm{Tl}-\mathrm{Bb}$ and carbonate object V3V4). Oilfield Engineering, 2018, no. 2, pp. 26-32. In Rus.

20. Galkin V.I., Ponomareva I.N., Koltyrin A.N. Development of probabilistic and statistical models for evaluation of the effectiveness of proppant hydraulic fracturing (on example of the $\mathrm{Tl}-\mathrm{Bb}$ reservoir of the Batyrbayskoe field). Bulletin of Perm National Research Polytechnic University. Geology. Oil \& Gas engineering \& Mining, 2018, no. 1, pp. 37-45. In Rus.

Received: 18 December 2018. 